\title{
Escherichia coli enteropatogênica: uma categoria diarreiogênica versátil
}

\author{
Enteropathogenic Escherichia coli: a versatile diarrheagenic category \\ Escherichia coli enteropatógena: una categoría diarreogénica versátil
}

Cintya de Oliveira Souza

Instituto Evandro Chagas/SVS/MS, Ananindeua, Pará, Brasil

Thainara Roberta Barros Melo

Laboratório de Patologia Clínica Dr. Paulo C. de Azevedo, Belém, Pará, Brasil

Caroline do Socorro Barros Melo

Instituto de Ciências Biológicas, Universidade Federal do Pará, Belém, Pará, Brasil

\author{
Êmily Moreira Menezes \\ Hospital Israelita Albert Einstein, São Paulo, São Paulo, Brasil \\ Aline Correa de Carvalho \\ Faculdade Carajás, Marabá, Pará, Brasil \\ Leni Célia Reis Monteiro \\ Instituto Evandro Chagas/SVS/MS, Ananindeua, Pará, Brasil
}

\section{RESUMO}

A Escherichia coli enteropatogênica (EPEC) foi a primeira categoria de E. coli reconhecida como diarreiogênica e ainda hoje está associada a casos esporádicos e surtos de diarreia infantil. Em 1995, a EPEC foi classificada em típica e atípica e, até $\mathrm{o}$ momento, muito se tem pesquisado sobre as diferenças patogênicas e epidemiológicas destas duas subcategorias e sua similaridade com outras categorias. Para consolidar estas informações, a presente pesquisa avaliou 98 fontes bibliográficas, sendo 81 artigos, oito teses, quatro dissertações e cinco livros. Essa pesquisa destacou os seguintes resultados e conclusões: as EPEC típicas (EPEC-t) têm como principal reservatório os seres humanos, no entanto já foram registradas raras ocorrências em alguns animais silvestres; as EPEC atípicas (EPEC-a) são encontradas entre humanos e uma variedade de outros hospedeiros animais que podem servir de reservatório e de fonte de contaminação para o homem e o ambiente, além disso, as EPEC-a apresentam inúmeros fatores de virulência comuns e específicos de outras categorias patogênicas, sugerindo que o aumento de sua prevalência esteja relacionado ao fenômeno de interconversão; a presença da região LEE (locus of enterocyte effacement) completa (LEEA-D) e da ilha de patogenicidade OI-122 (efa 7/lifA, nleB, nleE, set/ent), juntamente com os genes da hemolisina (ehxA) e da adesina (paa) podem auxiliar na identificação de potenciais estirpes patogênicas de EPEC-a; a identificação conclusiva de EPEC é realizada pelo diagnóstico molecular, onde se pesquisam os genes eae, EAF e stx, sendo o perfil eae +EAF + stx - de EPEC-t e o eae+EAF-stx- de EPEC-a.

Palavras-chave: Escherichia coli enteropatogênica; Epidemiologia; Fatores de Virulência.

\section{INTRODUÇÃO}

A Escherichia coli é uma bactéria pertencente à família Enterobacteriaceae, sendo amplamente distribuída na natureza, tendo como principal habitat o trato intestinal humano e animal ${ }^{1,2,3,4,5}$. A E. coli comensal, que faz parte da microbiota intestinal, não é patogênica e apresenta um importante papel fisiológico para o funcionamento do organismo. Existem seis categorias patogênicas de E. coli que causam infecção

\footnotetext{
Correspondência / Correspondence / Correspondencia:

Cintya de Oliveira Souza

Instituto Evandro Chagas, Seção de Bacteriologia e Micologia

Rodovia BR 316, km 7, s/n. Bairro: Levilândia

CEP: 67030-000 Ananindeua-Pará-Brasil

Tel.: +55 (91) 3214-2116 Fax: +55 (91) 3214-2114

E-mail: cintyaoliveira@iec.pa.gov.br
}

intestinal em homens e animais, sendo denominadas de E. coli diarreiogênicas ${ }^{6}$ que são diferenciadas pela presença de fatores de virulência como adesinas fimbriais e afimbriais, toxinas e invasinas, e classificadas em: E. coli enteropatogênica (EPEC), E. coli enterotoxigênica (ETEC), E. coli enteroinvasora (EIEC), E. coli enterohemorrágica (EHEC) ou E. coli produtora da toxina de Shiga (STEC), E. coli enteroagregativa (EAEC) e E. coli aderente difusa (DAEC) $)^{7,8,9}$.

A EPEC foi a primeira E. coli descoberta em 1940 e ainda hoje é considerada a mais versátil entre as categorias diarreiogênicas e uma das principais causas de diarreia em crianças menores de 5 anos de idade $10,11,12,13$. Em 1995, a EPEC foi classificada em duas subcategorias: EPEC típica (EPEC-t) e atípica (EPEC-a). As EPEC-† são identificadas pela presença do gene eae (EPEC attaching and effacing) e plasmídio EAF (EPEC adherence factor). As EPEC-a apresentam 
o gene eae, porém são desprovidas do plasmídio EAF. Tanto EPEC-† como EPEC-a devem ser desprovidas do gene stx (Shiga toxina) que caracteriza a STEC/EHEC ${ }^{14}$. Por muito tempo, as EPEC-t estiveram associadas à diarreia infantil, mas atualmente observa-se uma redução desta subcategoria e o aumento de isolamento de EPEC-a ${ }^{15,16,17}$. Cada subcategoria é representada por diferentes sorotipos e as EPEC- $\dagger$ pertencem aos sorotipos: $\mathrm{O} 55: \mathrm{H}[6], \mathrm{O} 86: \mathrm{H} 34, \mathrm{O} 111: \mathrm{H}[2], \mathrm{O} 114: \mathrm{H} 2$, O119:H[6], O127:H6, O142:H6 e O142:H34, enquanto que as EPEC-a pertencem aos sorotipos: O26:H[11], O55:H[7], O55:H[34], O86:H[8], O111ac:H[8], O111:H[9], O111:H25, O119:H2, 0125:H6 e O128:H2, sendo identificados mais de 200 sorotipos de EPEC-a $a^{18}$.

A EPEC-† representa a categoria original de EPEC com comportamento epidemiológico restrito aos seres humanos e patogenia bem definida. A EPEC-a está associada à diarreia humana tanto em países desenvolvidos quanto em desenvolvimento, pode ser encontrada em diversos animais, alimentos e estar distribuída em diferentes ambientes tanto aquáticos como terrestres $2,3,5,19$. Apesar de sua ampla distribuição e da sua comprovada participação como causa de diarreia humana, sua patogênese e via de transmissão não estão claramente estabelecidas ${ }^{20}$.

mecanismo da patogênese de EPEC é a lesão A/E (attaching and effacing) que envolve genes localizados na região LEE (locus of enterocyte effacement), considerada uma ilha de patogenicidade, onde são encontrados os genes ler (regulador transcricional), esp (proteínas do sistema de secreção do tipo III), tir (translocated intimin receptor - receptor de intimina) e o gene eae/E. coli attachment-effacement (adesina intimina) ${ }^{21,22}$. Três estágios de interação entre EPEC e a célula podem ser observados: 1) aderência localizada ( $\mathrm{AL}$ ) mediada pela fímbria BFP (bundle-forming pilus) e codificada pelo gene plasmidial $E A F$; 2) sinais de transdução; e 3) aderência íntima promovida pela intimina (gene eae) ${ }^{7}$.

Para o diagnóstico da EPEC, inicialmente realiza-se a coprocultura para $\mathrm{O}$ isolamento da bactéria que depois será submetida a diferentes testes complementares como: sorotipagem, ensaio de aderência com células HEp-2, prova de FAS (fluorescent actin-staining - coloração fluorescente à actina) e técnicas de biologia molecular (reação em cadeia mediada pela polimerase - PCR e suas variações) que amplificam genes que codificam os fatores de virulência que permitem a identificação e subclassificação de EPEC. Outros fatores de virulência que não estão envolvidos com a classificação desta categoria podem estar presentes e distribuídos entre EPEC- $\dagger$ e EPEC- $a^{19,22,23}$

Diante das constantes pesquisas e da diversidade de questionamentos em relação à EPEC, este artigo apresenta uma consolidação de material bibliográfico publicado nas últimas duas décadas, com objetivo de proporcionar aos alunos, profissionais microbiologistas, epidemiologistas, médicos e outros da área biomédica, esclarecimentos sobre a epidemiologia, os principais fatores de virulência e a identificação laboratorial desta importante e versátil categoria de E. coli diarreiogênica e suas subcategorias.

\section{METODOLOGIA}

Esta revisão consiste em uma pesquisa bibliográfica exploratória realizada em duas bases de dados científicas: Medical Literature Analysis and Retrieval System Online (Medline/PubMed) e Scientific Electronic Library Online (SciELO). Além dos artigos, teses, dissertações e livros didáticos referentes à área microbiológica foram utilizados como fontes bibliográficas. A estratégia de busca nas bases de dados foi realizada por meio da combinação dos termos "EPEC", "EPEC típica", "EPEC atípica" com os seguintes preditores em português, inglês e espanhol, "fatores de virulência", "patogenicidade", "humanos", "animais", "ambiente", "diagnóstico" e "epidemiologia". $\bigcirc$ período considerado na busca bibliográfica foi de 1992 a 2013. O critério de busca permitiu a identificação de aproximadamente 400 publicações, que foram inicialmente avaliadas quanto ao título e resumo, sendo selecionadas as que abordavam a combinação dos termos "EPEC", "EPEC típica", "EPEC atípica" com os preditores citados acima. Posteriormente, uma leitura completa das publicações pré-selecionadas foi realizada e o fator relevante para a seleção final dos artigos foi quando o conjunto composto por título, resumo, resultado e discussão forneciam informações para o esclarecimento da epidemiologia, dos principais fatores de virulência e da identificação laboratorial de E. coli enteropatogênica e suas subcategorias. Assim, 98 publicações foram utilizadas nesta revisão, sendo 81 artigos, oito teses, quatro dissertações e cinco livros ou capítulos de livro. Para síntese desta revisão, os resultados foram apresentados de maneira condensada na forma de quadros.

\section{RESULTADOS}

As EPEC podem ser isoladas de diferentes fontes como em: alimentos crus e processados; animais domésticos e silvestres; ambientes naturais como água, solo e areia de praia; fezes e solo de abatedouro bovino e fazendas. Observa-se, com os resultados deste levantamento, que a maioria dos artigos sobre epidemiologia não difere as EPEC em subcategoria típica ou atípica. Entre os trabalhos que fizeram esta diferenciação, pode-se observar que as EPEC-a são, constantemente, identificadas na água e com frequência em animais domésticos e silvestres, enquanto que as EPEC- $\dagger$ foram identificadas em raras ocorrências entre animais silvestres (como macaco de cativeiro, coiote e cães) e, no ambiente aquático natural, como rios, lagoas e enseadas. Com relação à frequência em humanos, tanto a EPEC-a quanto a EPEC- $\dagger$ foram identificadas em crianças e adultos com diarreia aguda ou sem diarreia. Também foram identificadas poucas ocorrências de infecção concomitante com outros micro-organismos (Quadro 1). 


\begin{tabular}{|c|c|c|}
\hline $\begin{array}{l}\text { Fontes de } \\
\text { isolamento }\end{array}$ & $\begin{array}{c}\text { Descrição da fonte de isolamento de EPEC } 1,{\text { EPEC }-t^{2}}^{2}, \\
\text { EPEC-a }{ }^{3}\end{array}$ & Referências \\
\hline Alimento & $\begin{array}{l}\text { Linguiça toscana', leite pasteurizado', carne moída }{ }^{1} \text {, } \\
\text { alface }^{1} \text {, tomate }{ }^{1} \text {, água de consumo }{ }^{3} \text {, leite de ovelha }{ }^{3}\end{array}$ & $\begin{array}{l}\text { FRANCO }{ }^{24} \text {; SILVA et al }{ }^{25} \text {; BERBARDI et a }{ }^{26} \text {; MARTINS et a }\left.\right|^{27} \text {; GÓMEZ- } \\
\text { ALDAPA et } \mathrm{al}^{28} \text {; CANIZALEZ-ROMAN et al }\left.\right|^{29} ; \text { OTERO et } \mathrm{al}^{30}\end{array}$ \\
\hline Animal & $\begin{array}{l}\text { Cães }^{1,2,3}, \text { gatos }^{1} \text {, bezerros } \\
\end{array}$ & 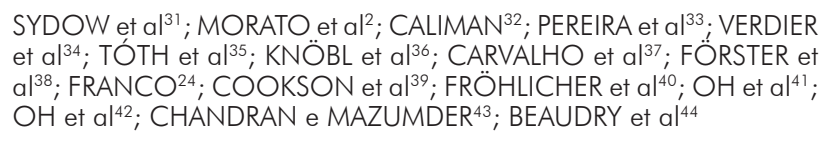 \\
\hline Ambiente & $\begin{array}{l}\text { Areia de praia }{ }^{1} \text {, rios, lagoas e enseadas }{ }^{2,3}, \text { água de }^{1} \\
\text { lastro e regióes portuárias }{ }^{3} \text {, água de consumo } 0^{1} \text {, fezes } \\
\text { bovinas e solo (fazenda e abatedouro) }{ }^{3}, \text { solo', água } \\
\text { de irrigação' }\end{array}$ & 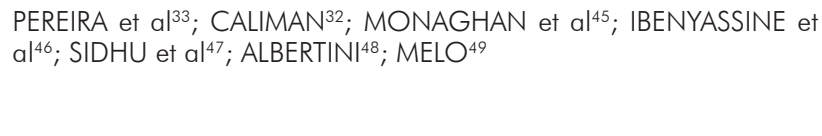 \\
\hline Humano & Crianças e adultos com ou sem diarreia ${ }^{1,2,3}$ & 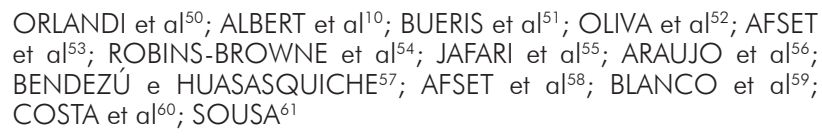 \\
\hline
\end{tabular}

Quadro 1 - Fontes de isolamento de EPEC

Entre os principais fatos epidemiológicos envolvendo as EPEC, observa-se: a) o aumento da ocorrência em EPEC-a e diminuição de EPEC-t entre amostras de fezes humanas, sendo relatados surtos de diarreia causada por EPEC-a em diversos países; b) a presença de EPEC em animais domésticos evidenciando esses animais como possíveis reservatórios; e c) a classificação de EPEC-a em dois subgrupos com base na classificação sorológica (Quadro 2).
Aumento da frequência de EPEC-a e diminuição de EPEC-† entre amostras humanas (fezes)

Surtos de EPEC-a em humanos

Gatos diarreicos ou saudáveis servem de reservatório para infecções por EPEC* em humanos

Cães aparentemente sadios, sem sintomas de colibacilose, podem servir como reservatório de EPEC* para humanos

Cepas de EPEC-a podem ser classificadas em dois subgrupos: pertencentes a sorotipos clássicos, associados com diarreia; e as que são não tipáveis ou pertencem aos sorotipos não clássicos
AFSET et $a^{158}$; BUERIS et $a^{151}$; COSTA et a ${ }^{60}$; AFSET et a $\left.\right|^{53}$; SHETTY ef $\mathrm{a}^{62}$; HANNAOUI et al ${ }^{63} ;$ SPANO et al ${ }^{64} ; \mathrm{VILCHEZ} \mathrm{et} \mathrm{al}{ }^{65} ;$ FRANZOLIN et $\mathrm{al}^{166}$; TRABULSI et $\mathrm{al}^{14}$

SAKKEJHA et $\mathrm{al}^{67}$; SHETTY et $\mathrm{al}^{62} ; \mathrm{HANNAOUI}$ et $\mathrm{al}^{63}$; SPANO et $\mathrm{al}^{64}$; VILCHEZ et al ${ }^{65}$; FRANZOLIN et al ${ }^{160}$

MORATO et al ${ }^{2}$

SYDOW et al ${ }^{31}$

SCALETSKY et al ${ }^{68}$

Sem diferenciação entre EPEC típica e atípica.

Quadro 2 - Principais fatos epidemiológicos envolvendo EPEC 


\begin{tabular}{|c|c|c|c|}
\hline $\begin{array}{l}\text { Gene ou } \\
\text { região de } \\
\text { virulência }\end{array}$ & Especificação do fator de virulência & $\begin{array}{c}\text { Frequência de } \\
\text { EPEC atípica } \\
\text { No/N }^{0 *}(\%)^{* *}\end{array}$ & Referências \\
\hline hly & Alfa-hemolisina & $31 / 99(31,3)$ & \multirow{2}{*}{ GOMES et al ${ }^{69}$} \\
\hline \multirow{2}{*}{ irp2 } & \multirow{2}{*}{ Gene que codifica uma proteína biosintética chamada Yersinia spp. } & $31 / 99(31,3)$ & \\
\hline & & $12 / 57(21,1)$ & AFSET et al70 \\
\hline \multirow{3}{*}{ ehxA/E-hly } & \multirow{3}{*}{ Hemolisina de EHEC (EHEC hemolysin) } & $1 / 38(3,0)$ & DULGUER et $\mathrm{a}^{71}$ \\
\hline & & $8 / 57(14,0)$ & AFSET et a ${ }^{70}$ \\
\hline & & $15 / 126(11,9)$ & SCALETSKY et al ${ }^{68}$ \\
\hline ure $D$ & Proteína UreD associada à urease & $7 / 57(12,3)$ & \multirow{3}{*}{ AFSET et al ${ }^{70}$} \\
\hline nleC & Non-LEE effector protein C & $50 / 57(87,7)$ & \\
\hline nleF & Non-LEE effector protein $F$ & $40 / 57(70,2)$ & \\
\hline \multirow{2}{*}{$n l e B$} & \multirow{2}{*}{ Non-LEE effector protein $B$} & $46 / 126(36,5)$ & SCALETSKY et al ${ }^{68}$ \\
\hline & & $23 / 57(40,4)$ & AFSET et al ${ }^{70}$ \\
\hline \multirow{2}{*}{ nleE } & \multirow{2}{*}{ Non-LEE effector protein $E$} & $46 / 126(36,5)$ & SCALETSKY et al ${ }^{68}$ \\
\hline & & $23 / 57(40,4)$ & AFSET et al70 \\
\hline \multirow{2}{*}{ efal/lifA } & \multirow{2}{*}{$\begin{array}{l}\text { Fator de aderência de EHEC (EHEC factor for adherence)/ } \\
\text { lymphocyte inhivitory factor A }\end{array}$} & $38 / 126(30,1)$ & SCALETSKY et al ${ }^{68}$ \\
\hline & & $17 / 57(28,8)$ & AFSET et al70 \\
\hline afa & Adesina afimbrial (afimbrial adhesins) & $9 / 126(7,1)$ & \multirow{3}{*}{ SCALETSKY et al ${ }^{68}$} \\
\hline ipf & Fímbria LPF (long polar fimbriae) & $34 / 126(27,0)$ & \\
\hline \multirow{2}{*}{ paa } & \multirow{2}{*}{$\begin{array}{l}\text { Proteína suína associada à lesão A/E (porcine attaching and effacing } \\
\text { lesion-associated protein) }\end{array}$} & $24 / 126(19,0)$ & \\
\hline & & $23 / 57(40,4)$ & AFSET et al70 \\
\hline \multirow{2}{*}{ sen ou set } & \multirow{2}{*}{ Enterotoxina similar a ShET de Shigella flexneri (ShET enterotoxin) } & $28 / 126(22,2)$ & SCALETSKY et al ${ }^{68}$ \\
\hline & & $23 / 57(40,4)$ & AFSET et al ${ }^{70}$ \\
\hline \multirow{4}{*}{ ast $A$ ou east- 1} & \multirow{4}{*}{ Toxina termoestável (enteroaggregative heat-stable toxin - EAST-1) } & $42 / 126(33,3)$ & SCALETSKY et al ${ }^{68}$ \\
\hline & & $29 / 99(29,3)$ & GOMES et a ${ }^{69}$ \\
\hline & & $8 / 57(14,0)$ & AFSET et al70 \\
\hline & & $29 / 65(44,6)$ & \multirow{4}{*}{ DULGUER et $\mathrm{al}^{71}$} \\
\hline per & Regulador codificado por plasmídeo (plasmid-encoded regulator) & $33 / 65(50,7)$ & \\
\hline LEEA-D & $\begin{array}{l}\text { Sequências A-D que fazem parte de LEE (locus of enterocyte } \\
\text { effacement) }\end{array}$ & $65 / 65(100,0)$ & \\
\hline tir & Receptor de intimina & $64 / 65(98,4)$ & \\
\hline $\operatorname{shf}$ & Fator de virulência similar a Shigella flexneri & $2 / 57(3,5)$ & \multirow{2}{*}{ AFSET et al ${ }^{70}$} \\
\hline pet & Enterotoxina codificada por plasmídeo (plasmid encoding toxin) & $1 / 57(1,8)$ & \\
\hline$c d t$ & Toxina citoletal distendida (cytolethal distending toxin) & $2 / 126(1,6)$ & SCALETSKY et al ${ }^{68}$ \\
\hline
\end{tabular}

* Número de amostras positivas / número de amostras pesquisadas; ** Percentual de amostras positivas.

Quadro 3 - Fatores de virulência observados em EPEC atípicas 
De acordo com o quadro 3, observa-se que os genes da região LEEA-D que codificam o sistema de secreção do tipo III, as proteínas secretadas por este sistema (Esp) e a intimina (eae-EPEC attaching and effacing), e o gene tir que codifica o receptor de intimina, ambos participantes da lesão $A / E$, foram os genes identificados em EPEC-a com as maiores frequências, $100 \%$ e 98,4\%, respectivamente. Os demais fatores de virulência observados em EPEC-a são as proteínas efetoras não codificadas pelo LEE- (non LEE-nle), nle C $(87,7 \%)$ e $F(70,2 \%)$, seguido de nle-B e $E$ (ambas com variação de frequência entre 36,5\% a $40,4 \%$ ) e o gene per (regulador de LEE localizado em um plasmídeo) com 50\%. As frequências aproximadas de $40 \%$ a $20 \%$ foram observadas para os fatores $h l y$, irp2, efa/lifA, ipf, paa e sen. Frequências abaixo de $20 \%$ foram observadas para os demais fatores de virulência (ehxA, afa, shf, pet e cdt). Especificamente para enterotoxina EAST-1, codificada pelo gene astA e pesquisada por vários autores, os percentuais variaram de $14 \%$ a $44,6 \%$. Todos esses fatores não relacionados à lesão A/E de EPEC (hly, irp2, efa/lifA, ipf, paa, sen, ehxA, afa, shf, pet e cdt) são frequentemente encontrados em outras categorias patogênicas como $E$. coli uropatogênica (UPEC), EHEC, EAEC, DAEC, além das EPEC.

De acordo com a revisão bibliográfica, podem-se identificar quatro técnicas laboratoriais utilizadas para o diagnóstico e caracterização de EPEC: a sorotipagem, o teste de adesão em cultura de células, o FAS e as técnicas de biologia molecular. Entre estas técnicas, as únicas utilizadas para diferenciação entre EPEC-† e EPEC-a, são as técnicas de biologia molecular como a PCR convencional, PCR multiplex e PCR em tempo real (Quadro 4). Estas técnicas laboratoriais abrangem metodologias voltadas para aspectos específicos da EPEC como a identificação de antígenos (sorotipagem) e genes codificantes de fatores de virulência (PCR e suas variações), bem como, aspectos voltados à investigação da interação da bactéria com as células, como a formação da lesão $A / E$ e condensamento de actina constatados no teste de FAS e a observação e classificação do tipo de adesão que as EPEC promovem em cultura de células.

A identificação e a diferenciação entre EPEC-† e EPEC-a compreendem, principalmente, critérios moleculares como a pesquisa dos genes eae, stx, bfp e a sequência $E A F$, sendo o perfil eae + stx $-E A F+b f p+$ identificado para EPEC-t e o perfil eae+stx-EAFbfp-l+ para EPEC-a. Aspectos importantes que contribuem para melhor caracterização de EPEC-† e EPEC-a são: a observação da formação da lesão $A / E$ que é encontrada nestas duas subcategorias; a expressão de BFP, o tipo de aderência promovido em cultura de células, sendo o padrão único de $\mathrm{AL}$ encontrado nas EPEC-t; e padrões variados encontrados entre as EPEC-a (Quadro 5).

\begin{tabular}{|c|c|c|c|}
\hline Técnica laboratorial & $\begin{array}{l}\text { Diferenciação } \\
\text { entre EPEC- }+ \text { e } \\
\text { EPEC-a }\end{array}$ & Descrição & Referências \\
\hline Sorotipagem & Não & $\begin{array}{l}\text { Identificação de antígenos } \\
\text { somáticos, flagelares e capsulares }\end{array}$ & LLUQUE et al ${ }^{72}$; GILLESPIE ${ }^{73}$; OPLUSTIL et al ${ }^{23} ;$ FRANCO $^{24}$ \\
\hline $\begin{array}{l}\text { FAS (fluorescent actin } \\
\text { staining) }\end{array}$ & Não & $\begin{array}{l}\text { Detecção do condensamento } \\
\text { de actina identificado durante a } \\
\text { formação da lesão A/E }\end{array}$ & LLUQUE et $\mathrm{al}^{72} ; \mathrm{VIDAL}$ et $\mathrm{al}^{19} ;$ MOREIRA et $\mathrm{al}^{74}$; PAIVA $^{75}$ \\
\hline $\begin{array}{l}\text { Aderência em culturas de } \\
\text { células }\end{array}$ & Não & $\begin{array}{l}\text { Observação e classificação do tipo } \\
\text { de aderência }\end{array}$ & 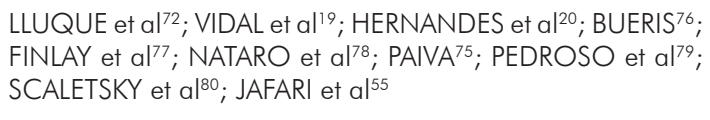 \\
\hline $\begin{array}{l}\text { Biologia molecular (PCR } \\
\text { convencional, PCR multiplex } \\
\text { e PCR em tempo real) }\end{array}$ & Sim & $\begin{array}{l}\text { Identificação de genes codificantes } \\
\text { de fatores de virulência }\end{array}$ & 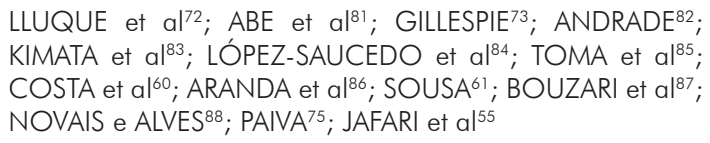 \\
\hline
\end{tabular}

Quadro 4 - Técnicas para identificação e diferenciação de EPEC típica e atípica

\begin{tabular}{|c|c|c|}
\hline Característica & EPEC- $\dagger$ & EPEC-a \\
\hline Gene eae (E. coli attachment-effacement) & + & + \\
\hline Genes stx (Shiga toxin) & - & - \\
\hline Sequência EAF (EPEC adherence factor) & + & - \\
\hline Gene bfpA (bundle-forming pilus) & + & $-/+^{*}$ \\
\hline Formação da lesão A/E (attaching and effacing) & + & + \\
\hline Expressão de BFP (bundle-forming pilus) & + & - \\
\hline Aderência padrão** & $\mathrm{AL}$ & LAL/DA/AA/AL ${ }^{* * *} N A$ \\
\hline
\end{tabular}

+: Presença; -: Ausência; * Sequência do bfp incompleta (Bortolini et a ${ }^{89}$ ); ${ }^{* *}$ Aderência padrão em células HeLa/HEp-2 - LA: aderência localizada; LAL: LA like; DA: aderente difusa; AA: aderência agregativa; NA: não aderente; ${ }^{* * *}$ Aderência localizada na EPEC-a é independente do bfp.

Quadro 5 - Características importantes de EPEC-† e EPEC-a que auxiliam na identificação laboratorial 


\section{DISCUSSÃO}

A EPEC é uma das principais causadoras de enteroinfecções em crianças e adultos e, nas últimas décadas, estudos sobre esta categoria apontam que esse patógeno está cada vez mais presente na vida de diversas populações, interagindo e se adaptando ao ambiente, ao ponto de serem classificadas em duas subcategorias distintas: EPEC- $\dagger$ e EPEC- $a^{43,48,53,68}$

As EPEC- $\dagger$ estiveram por longo tempo associadas à diarreia infantil, no entanto, o atual cenário etiológico demonstra redução na prevalência desta subcategoria em detrimento do aumento dos isolamentos de EPEC-a em casos de diarreia ${ }^{15,16,17}$, inclusive como única categoria de $E$. coli diarreiogênica associada à diarreia ${ }^{90}$. Além deste fato, constatou-se que recentes surtos ocorridos em humanos em diferentes localidades foram ocasionados por EPEC-a ${ }^{51,53,58,62,63}$.

Além dos seres humanos, as EPEC podem ser identificadas em uma diversidade de animais e ambientes naturais, porém este comportamento epidemiológico pode ser diferenciado dependendo da subcategoria de EPEC. Segundo Oh et al ${ }^{41,42}$, Tóth et $a^{35}$, Ibenyassine et $a^{46}$ e Chandran e Mazumder ${ }^{43}$, as EPEC-a são identificadas com frequência em águas de rios, enseadas, lagoas e regiões portuárias, além de estarem presentes em animais domésticos e silvestres. Os mesmos estudos apontam a presença das EPEC- $t$ nos mesmos ambientes relatados acima, porém, sua ocorrência entre os animais é rara, tendo sido identificadas apenas em animais silvestres criados em cativeiro, como o macaco ${ }^{37}$, coiotes e cães de uma área de bacia hidrográfica no Canadá ${ }^{43}$. Estes dados apontam para uma diferença comportamental entre as duas subcategorias que, provavelmente, influenciam $O$ modo de transmissão para o ser humano.

Os artigos de revisão de Hernandes et $a^{20}$ e Sousa ${ }^{91}$, ressaltam o alto índice de isolados de EPEC-a em animais domésticos e silvestres, embora não haja evidência de transmissão direta de animais para seres humanos, algumas estirpes de EPEC-a isoladas de animais pertencem aos sorogrupos implicados em doenças humanas, por exemplo O26, O103, O119, 0128 e 0142 .

Ao longo de muitos anos, o homem tem mantido um estreito contato com os animais, porém esta convivência tem se intensificado, principalmente em relação aos animais domésticos e, apesar de cães e gatos terem sido animais de companhia por milhares de anos, nos dias atuais desempenham um importante papel para a sociedade humana ${ }^{32}$. $\bigcirc$ problema dessa convivência é que tanto os animais domésticos quanto os seus donos tornam-se possíveis reservatórios de doenças como gastroenterite humana ${ }^{33}$. Um importante achado epidemiológico sobre as EPEC-a é a presença delas em animais domésticos saudáveis ou diarreicos 2,31 , aumentando as chances de transmissão para humanos, mas mesmo com sua ampla distribuição e sua comprovada participação como causa de diarreia humana, sua via de transmissão não está claramente estabelecida ${ }^{20}$.

Em contraste com a epidemiologia das EPEC-a, Hernandes et $a^{20}$ e Sousa ${ }^{91}$ descrevem $O$ ser humano como principal reservatório de EPEC-t, sendo raramente identificada entre os animais. Esta observação está de acordo com outros estudos que consideram os seres humanos como único reservatório de EPEC-t, com exceção de alguns relatos de amostras isoladas de $\operatorname{cães}^{44}$ e macacos ${ }^{37}$.

Estudos epidemiológicos que demonstram a frequência destes patógenos em diferentes animais e no ambiente são importantes para revelar que as EPEC-a, assim como a EHEC (categoria zoonótica de E. coli), podem ser observadas em diferentes hospedeiros animais e indicam que eles podem ser um reservatório destes patógenos com potencial para contaminar as águas superficiais e impactar na saúde humana ${ }^{43}$. No caso específico de EPEC-a, esse impacto vai depender do perfil de virulência apresentado pela bactéria. Como também demonstrado por Chandran e Mazumder ${ }^{43}$, a maioria das amostras de EPEC isoladas de animais foram de EPEC-a (60/593: 10,11\%), comparado ao número inferior de EPEC-† (8/593: 1,3\%) isoladas apenas de coiotes (sete isolados) e cães (um isolado).

Gomes et $a^{69}$, ao avaliarem 17 sequências gênicas relacionadas a fatores de virulência em EPEC-a (eae+EAF-), identificaram 34 diferentes perfis de virulência sendo os mais frequentes: eae $(31,3 \%$ $31 / 99)$, eae hly astA pet irp2 $(8,1 \%-8 / 99)$, eae hly $(6,1 \%-6 / 99)$, eae shf $(5,1 \%-5 / 99)$, eae irp2 $(5,1 \%$ - 5/99), eae perA bfpA astA (4\% - 4/99), eae perA bfpA $(4 \%$ - 4/99). Além destes fatores de virulência citados por Gomes et $a^{69}$, Dulguer et $a^{71}$, incluindo em sua pesquisa os fatores de virulência comuns em EPEC (lesão $A / E)$, como a região LEE (A-D), o receptor de intimina (Tir) e o gene regulador plasmidial (per), identificaram os perfis de virulência: LEEA-D tir $(43 \%$ - 28/65), perA LEEA-D tir astA (33,8\% - 22/65), perA LEEA-D tir $(13,8 \%$ - 9/65) como os mais frequentes, observando que, com exceção da toxina AST, os demais fatores de virulência pesquisados estiveram ausentes (hly, pet, afa, ehxA, agg-A). Estes dados indicam, com exceção dos fatores de virulência comuns à lesão $A / E$, que ocorre variação e flexibilidade dos fatores de virulência acessórios presentes em EPEC-a.

Apesar do gene astA (enterotoxina EAST-1) ser detectado com frequência em EPEC-a (Quadro 3) quando comparada à EPEC- $t^{71}$ e dos surtos de diarreia relacionados à EPEC-a produtora de EAST-1 (gene astA), Yatsuyanagi et $a^{92}$ e Afset et $a^{70}$ observaram associação negativa deste gene (astA) e de outros fatores de virulência (yjaA, ibeA e b 1121) com EPEC-a isoladas de casos diarreicos. De acordo com Scaletsky 
et $a^{68}$, foram identificados dois marcadores de virulência, os genes para toxina ehxA e a adesina paa, que poderão ser úteis na detecção de EPEC-a e que foram associados positivamente com EPEC-a isoladas de crianças com diarreia ${ }^{70}$.

Além de ehxA e paa, os genes localizados na ilha de patogenicidade Ol-122 (efal/lifA, nleB, nleE, set/ent) quando tiveram suas frequências comparadas entre EPEC-a proveniente de casos com e sem diarreia, foram associados significativamente às amostras de EPEC-a isoladas de casos diarreicos ${ }^{70}$. De acordo com Vieira et $a^{193}$, a presença de ilha de patogenicidade (PAI) Ol122 e a ocorrência simultânea dos genes efal/lifA, sen, nleB e nleE foram estatisticamente associadas à EPEC-a isoladas de casos diarreicos, sugerindo que a detecção completa da PAI Ol122 poderia auxiliar na identificação de potenciais estirpes patogênicas de EPEC-a. Enquanto que a presença de PAI Ol122 é complementar à patogênese de EPEC-a, as EPEC-a que apresentam o locus LEE completo (A-D) possuem potencial patogênico ${ }^{71}$, o que foi confirmado por Trabulsi et $a^{14}$ ao observarem que 75\% das EPEC-a LEEA-D+ apresentam potencial para promover a lesão $A / E$ observado pelo teste de FAS positivo.

Entre as EPEC com perfil de virulência (eae+EAF$s t x-)$ classificadas como EPEC-a, Trabulsi et $a^{14}$ identificaram que a maioria (80\% - 72/90) apresentou $\mathrm{AL}$ ou suas variações como: a aderência localizada like (LAL) e a aderência localizada após $6 \mathrm{~h}$ de ensaio (LA6). No entanto, outros tipos de aderência como a aderência agregativa (AA) e aderência difusa (AD) e a combinação de $A L$ com $A A$ ou $A D$ também foram observadas. Isto indica que o teste de adesão em cultura de células não tem poder discriminatório para diferenciação entre EPEC- $\dagger$ e EPEC-a. $\bigcirc$ mesmo deve ser utilizado para complementar os estudos de patogenicidade para esta subcategoria.

De acordo com Elias et $a^{194}$, ao avaliarem a detecção de marcadores de virulência entre 34 E. coli pertencentes aos sorogrupos de EPEC apresentando o fenótipo AA, observaram que apenas duas amostras foram positivas para o gene eae, nas quais os demais marcadores pesquisados foram negativos (bfp, aggR, aaf, shf, astA e irp). Os marcadores mais frequentes entre as amostras eae negativas foram: aggR (35,3\%), shf $(55,9 \%)$, astA $(64,7 \%)$ e irp $(70,6 \%)$, característicos de EAEC, sugerindo que apesar das amostras de EPEC-a compartilharem diversos genes de virulência entre as outras categorias patogênicas, incluindo estes fatores frequentes entre EAEC, quando as E. coli apresentaram adesão $A A$, existiu baixa propabilidade das mesmas serem EPEC-a, contudo a adesão AA não excluiu uma $E$. coli de ser EPEC-a.

Um estudo com EPEC-a (eae+EAF-stx-) isoladas de pacientes com diarreia sanguinolenta, utilizando o MLST (multilocus sequence typing), os perfis de virulência e a sorotipagem, identificou estas amostras como EHEC que perderam o gene stx durante a infecção ${ }^{95}$. Este evento é conhecido como interconversão de $E$. coli $A / E$, ocasionado pelo ganho ou perda de genes por transferência horizontal ou por meio de elementos genéticos móveis, tornando mais complicada a caracterização das diferentes categorias de E. coli diarreiogênica, principalmente entre as produtoras de lesão $\mathrm{A} / \mathrm{E}$ que apresentam o gene eae+, como a EPEC e a STEC/EHEC96. Este evento pode influenciar os aspectos epidemiológicos de EPEC-a e justificar a capacidade de adquirir, manter e expressar genes de virulência codificados por outras categorias de E. coli diarreiogênicas ${ }^{97}$.

Nesta revisão foram identificadas quatro técnicas capazes de identificar E. coli enteropatogênica: a sorotipagem, o teste de adesão em cultura de células, - FAS (fluorescent actin staining) e as técnicas de biologia molecular $55,72,73,74,75,80$. De acordo com Lluque et $a^{72}$, são utilizados dois métodos principais: a sorotipagem e a PCR, sendo apenas esta última com potencial para identificação de EPEC. Atualmente a sorotipagem é uma técnica utilizada especificamente para detecção apenas de antígenos somáticos e flagelares, sendo inapropriada para identificação de EPEC e diferenciação das suas subcategorias, pois um mesmo sorogrupo ou sorotipo pode ser EPEC- $\dagger$ ou EPEC-a. Entretanto, essa técnica tem uma importância epidemiológica para descrição dos sorotipos por reservatórios ou regiões geográficas, além de permitir a classificação da EPEC-a em dois subgrupos: sorotipos clássicos, associados com diarreia e as que não são tipáveis pertencentes aos sorotipos não clássicos $23,24,68,73$

O diagnóstico molecular é capaz de identificar EPEC e diferenciar EPEC- $\dagger$ de EPEC-a e os genes alvos utilizados neste diagnóstico são: eae, que está presente nas duas subcategorias de EPEC; o gene bfp, presente apenas na EPEC-t; e os genes stx, que são ausentes entre as EPEC, pois são específicos de EHEC/ STEC (E. coli enterohemorrágica) ${ }^{20}$. Para identificação de EPEC-a é mais comum o perfil EAF-e bfp-, porém, algumas EPEC-a podem apresentar o gene bfp (EAF- e bfp + ), isso acontece devido à existência de um plasmídeo EAF defectivo não detectado pela PCR, o que leva, na maioria das vezes, à ausência de expressão da fímbria BFP detectada molecularmente ${ }^{81}$.

De acordo com Trabulsi et al $^{14}$, das 99 amostras EPEC consideradas atípicas (eae $+\mathrm{EAF}-\mathrm{stx}-$ ) e testadas para o gene bfp, 14,1\% (14/99) foram positivas, no entanto apenas duas destas expressaram a fímbria BFP e carreavam o gene perA, significando que as EPEC-a podem expressar gene bfp mesmo com ausência do plasmídeo EAF, porém não apresentam $\mathrm{AL}$ em $3 \mathrm{~h}$ de ensaio, como observado em EPEC típicas. Baseando-se nesta informação, as EPEC-a podem expressar fímbria $B F P$, porém sem apresentar $A L$, ou podem apresentar $\mathrm{AL}$ que independe da presença da fímbria BFP. As observações de Trabulsi et al $^{14}$, de que alguns sorotipos como O142:H6, O119:H2 e O128:H2 apresentam 
bfp com EAF defectivo (EAF-), sugerem que a melhor característica para distinção de EPEC- $\dagger$ e EPEC-a seria a produção ou não da fímbria BFP, respectivamente, todavia a expressão desta fímbria pode não estar associada ao padrão de adesão localizada característico de EPEC-t.

Após extensas análises genotípicas e fenotípicas, Vieira et $a^{98}$ concluíram que amostras de EPEC-a (eae+EAF-stx-) de sorogrupos não clássicos compreendem um grupo heterogêneo composto por EPEC-a, EAEC, DAEC e UPEC que adquiriram o locus LEE por transferência horizontal, ou representam EPEC- $t$ que perderam o plasmídeo EAF (ou parte dele), EHEC/STEC que perderam o stx, ou E. coli da microbiota normal que adquiriram o locus LEE. Estes achados demonstram os questionamentos para esta subcategoria e evidenciam a versatilidade deste patotipo, que apesar das inúmeras pesquisas ainda necessita de esclarecimentos.

\section{CONCLUSÃO}

A EPEC é reconhecida como uma das principais causas da diarreia desde 1940 e, até o momento, continua associada a casos esporádicos e surtos de diarreia infantil, porém apresentando distintos perfis de virulência que permitem classificá-las em duas subcategorias (EPEC- $\dagger$ e EPEC-a) que apresentam comportamento epidemiológico distinto.

As EPEC- + e EPEC-a podem ser encontradas entre humanos, animais e no ambiente, entretanto com algumas diferenças. As EPEC-a são frequentemente encontradas entre humanos e em uma variedade de hospedeiros animais, indicando que os mesmos podem servir de reservatórios e de fontes de contaminação para o homem e o ambiente. As EPEC-t têm como principal reservatório os seres humanos, no entanto já foram registradas raras ocorrências em alguns animais, como macaco, coiote e cachorro.

Os fatores de virulência mais frequentes em EPEC-a são os codificados por genes que participam da lesão $\mathrm{A} / \mathrm{E}$ localizados na região LEE (A-D) que codificam $O$ sistema de secreção do tipo III, as proteínas secretadas por este sistema (Esp) e a intimina (eae) e o repector de intimina (tir), além das proteínas efetoras não codificadas por LEE (nle). A presença da região LEE completa (LEEA-D) e da ilha de patogenicidade Ol-122 (efal/lifA, nleB, nleE, set/ent), juntamente com os genes da hemolisina (ehxA) e adesina (paa), podem auxiliar na identificação de potenciais estirpes patogênicas de EPEC-a.

As EPEC-a apresentam inúmeros fatores de virulência comuns e específicos de outras categorias patogênicas como UPEC, EHEC, EAEC e DAEC. Esse fenômeno conhecido como interconversão pode ser responsável pelo aumento da frequência epidemiológica de EPEC-a e justificar a habilidade das EPEC-a em expressar diferentes perfis de virulência.

A identificação conclusiva de EPEC é realizada pelo diagnóstico molecular, onde se pesquisam os genes eae, EAF e stx, sendo o perfil eae $+E A F+s t x-$ de EPEC- $\dagger$ e o eae+EAF-stx- de EPEC-a. A investigação da presença do gene bfp e a expressão desta fímbria são importantes para esclarecer a patogênese de EPEC-a e melhor definir estes critérios moleculares utilizados para classificação das duas subcategorias de EPEC.

\section{Enteropathogenic Escherichia coli: a versatile diarrheagenic category}

\section{ABSTRACT}

Escherichia coli (EPEC) was the first category of E. coli to be discovered and continues to be associated with sporadic cases and outbreaks of diarrhea in children. In 1995 it was classified as typical and atypical EPEC and, until the current moment, much has been studied about the differences of these two pathogenic and epidemiological subcategories and their similarity to other pathotypes. To better know these studies and consolidate this information, this literature evaluated 98 bibliographic sources, with 81 articles, eight theses, four dissertations and five books. This research highlighted the following findings and conclusions: the atypical EPEC (a-EPEC) are present in humans and a variety of animal hosts, suggesting that they may serve as a reservoir and source of infection for humans and the environment; typical EPEC (t-EPEC) has humans as the main reservoir, however, they have been recorded in some rare occurrences wildlife; a-EPEC have several common virulence factors and specific categories of other pathogens, suggesting that increased prevalence of EPEC is related to interconversion, the presence of the LEE (locus of enteracyte effacement) complete region (LEEA-D) and pathogenicity island HI-122 (efal/lifA, nleB, nleE, set/ent) with the hemolysin gene (ehxA) and the toxin (paa) can help in identification of potentially pathogenic a-EPEC, but the conclusive identification of EPEC is performed by molecular diagnosis, which identifies genes eae, EAF and stx, and the profile eae+EAF+stx- of t-EPEC and eae+EAF-stx- in a-EPEC.

Keywords: Enteropathogenic Escherichia coli; Epidemiology; Virulence Factors. 


\section{Escherichia coli enteropatógena: una categoría diarreogénica versátil}

\section{RESUMEN}

El Escherichia coli enteropatógeno (EPEC) fue la primera categoría de E. coli reconocida como diarreogénica, siendo además asociada a casos esporádicos y brotes de diarrea infantil. En 1995, la EPEC fue clasificada en típica y atípica y, hasta este momento, mucho se ha investigado sobre las diferencias patógenas y epidemiológicas de estas dos subcategorías y su semejanza con otras categorías. Para consolidar estas informaciones, la presente investigación evaluó 98 fuentes bibliográficas, siendo 81 artículos, ochos tesis, cuatro disertaciones y cinco libros. Esta investigación destacó los siguientes resultados y conclusiones: las EPEC típicas (EPEC-t) tienen como principal reservorio a los seres humanos, sin embargo se han registrado raras ocurrencias en algunos animales silvestres; las EPEC atípicas (EPEC-a) se encuentran entre humanos y una variedad de otros huéspedes animales que pueden servir de reservorio y de fuente de contaminación para el hombre y el ambiente, además, las EPEC-a presentan innumerables factores de virulencia comunes y específicos de otras categorías patógenas, lo que sugiere que el aumento de su prevalencia esté relacionado al fenómeno de interconversión; la presencia de la región LEE (locus of enterocyte effacement) completa (LEEA-D) y de la isla de patogenicidad Ol-122 (efal/lifA, nleB, nleE, set/ent), junto a los genes de la hemolisina (ehxA) y la adhesina (paa) pueden auxiliar en la identificación de potenciales estirpes patógenas de EPEC-a; la identificación conclusiva de EPEC se realiza por el diagnóstico molecular, en el cual se investigan los genes eae, EAF y stx, siendo el perfil eae+EAF+stx - de EPEC-t y el eae+EAF-stx- de EPEC-a.

Palabras clave: Escherichia coli enteropatógena; Epidemiologia; Factores de Virulencia.

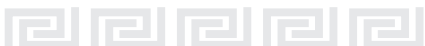

\section{REFERÊNCIAS}

1 Winn WJ, Alves S, Janda W, Koneman E, Procop G, Schrecherberger $P$, et al. Diagnóstico microbiológico: texto e atlas colorido. 6. ed. Rio de Janeiro: Guanabara Koogan; 2008. p. 208-25.

2 Morato EP, Leomil L, Beutin L, Krause G, Moura RA, Castro PAF. Domestic cats constitute a natural reservoir of human enteropathogenic Escherichia coli types. Zoonoses Public Health. 2009 Jun;56(5):229-37.

3 Moura RA. Estudo das relações clonais entre amostras de Escherichia coli atípica de origem animal e humana [tese]. São Paulo (SP): Universidade de São Paulo, Instituto de Ciências Biomédicas; 2009. 152 p.

4 Ayala CO. Sorologia de antígenos flagelares de amostras de Escherichia coli enteropatogênica EPEC e E. coli produtoras da toxina de Shiga (STEC) isoladas de diferentes animais e análise comparativa do gene fliC por PCR-RFLP [tese]. São Paulo (SP): Universidade de São Paulo, Instituto de Ciências Biomédicas; 2009. 62 p.

5 Silva N, Junqueira VCA, Silveira NFA, Taniwaki $M H$, Santos RFS, Gomes RAR. Manual de métodos de análise microbiológica de alimentos e água. São Paulo: Varela; 2010.

6 Martinez MB, Trabulsi LR. Enterobacteriaceae. In: Trabulsi LR, Alterthum F, editores. Microbiologia. São Paulo: Atheneu; 2008. p. 271-9.

7 Nataro JP, Kaper JB. Diarrheagenic Escherichia coli. Clin Microbiol Rev. 1998 Jan;1 1(1):142-201.
8 Teng LJ, Hsueh PR, Liaw SJ, Ho SW, Tsai JC. Genetic detection of diarrheagenic Escherichia coli isolated from children with sporadic diarrhea. J Microbiol Immunol Infect. 2004 Dec;37(6):327-34.

9 Nguyen TV, Le VP, Le HC, Gia KN, Weintraub A. Detection and characterization of diarrheagenic Escherichia coli from young children in Hanoi, Vietnam. J Clin Microbiol. 2005 Feb;43(2):755-60.

10 Albert MJ, Faruque SM, Faruque AS, Neogi PK, Ansaruzzaman M, Bhuiyan, et al. Controlled study of Escherichia coli diarrheal infections in Bangladeshi children. J Clin Microbiol. 1995 Apr;33(4):973-7.

11 Albert MJ. Epidemiology of enteropathogenic Escherichia coli infection in Bangladesh. Rev Microbiol. 1996;27(1):17-20.

12 Fagundes-Neto U, Scaletsky ICA. The gut at war: the consequences of enteropathogenic Escherichia coli infection as a factor of diarrhea and malnutrition. Sao Paulo Med J. 2000 Jan; 1 18(1):21-9.

13 Angeles GR. Principales características y diagnóstico de los grupos patógenos de Escherichia coli. Salud Publica Mex. 2002 sep;44(5):464-75.

14 Trabulsi LR, Keller R, Gomes TA. Typical and atypical enteropathogenic Escherichia coli. Emerg Infect Dis. 2002 May;8(5):508-13.

15 Souza EC, Martinez MB, Taddei CR, Mukai L, Gilio $A E$, Racz ML, et al. Perfil etiológico das diarreias agudas de crianças atendidas em São Paulo. Rev Pediatr. 2002 jan-fev;78(1):31-8. 
16 Ghosh PK, Ali A. Isolation of atypical enteropathogenic Escherichia coli from children with and without diarrhea in Delhi and the National Capital Region, India. J Med Microbiol. 2010 Oct;59(Pt 10): 11 156-62.

17 Moreno ACR, Fernandes Filho A, Gomes TAT, Ramos STS, Montemor LPG, Tavares VC, et al. Etiology of childhood diarrhea in the northeast of Brazil: significant emergent diarrheal pathogens. Diagn Microbiol Infect Dis. 2010 Jan;66(1):50-7.

18 Ochoa TJ, Contreras CA. Enteropathogenic Escherichia coli infection in children. Curr Opin Infect Dis. 2011 Oct;24(5):478-3

19 Vidal JE, Canizález RA, Gutiérrez J, Navarro F. Molecular pathogenesis, epidemiology and diagnosis of enteropathogenic Escherichia coli. Salud Publica Mex. 2007 Sep-Oct;49(5):376-86.

20 Hernandes RT, Waldir PE, Vieira MAM, Gomes AT. An overview of atypical enteropathogenic Escherichia coli. FEMS Microbiol Lett. 2009 Aug;297(2):137-49.

21 Dean P, Maresca M, Kenny B. EPEC's weapon of mass subversion. Curr Opin Microbiol. 2005 Feb;8(1):28-34.

22 Rocha LB. Desenvolvimento e padronização de testes imunocromatográficos para diagnóstico de Escherichia coli produtora da toxina de Shiga (STEC) e Escherichia coli enterotoxigênica (ETEC) [tese]. São Paulo (SP): Universidade de São Paulo, Instituto Butantan; 2012. 86 p.

23 Oplustil CP, Zoccoli CM, Tobouti NR, Sinto SI. Procedimentos básicos em microbiologia clínica. 3. ed. São Paulo: Sarvier; 2010. 402 p.

24 Franco RM. Escherichia coli: ocorrência em suínos abatidos na grande Rio e sua viabilidade experimental em linguiça frescal tipo toscana [tese]. Niterói (RJ). Universidade Federal Fluminense; 2002. 153 p.

25 Silva ZN, Cunha A, Lins MC, Carneiro LA, Almeida ACF, Queiroz MLP. Isolation and serological identification of enteropathogenic Escherichia coli in pasteurized milk in Brazil. Rev Saude Publica. 2001 Aug;35(4):375-9.

26 Bernardi E, Armas RD, Caldeira MF, Ribeiro GA. Caracterização microbiológica e sorológica de linhagens de Escherichia coli, isoladas de carne moída comercializada em Pelotas, RS. Hig Aliment. 2004 out; 18(125):82-6.

27 Martins AGLA, Nascimento AR, Mouchrek Filho JE, Mendes Filho NE, Souza AG, Aragão NE, et al. Atividade antibacteriana do óleo essencial do manjericão frente a sorogrupos de Escherichia coli enteropatogênica isolados de alfaces. Cienc Rural. 2010 ago;40(8):1791-6.
28 Gómez-Aldapa CA, Torres-Vitela MR, AcevedoSandoval OA, Rangel-Vargas E, Villarruel-López A, Castro-Rosas A. Presence of Shiga toxinproducing Escherichia coli, enteroinvasive E. coli, enteropathogenic E. coli, and enterotoxigenic E. coli on tomatoes from public markets in Mexico. J Food Prot. 2013 Sep;76(9): 1621-5.

29 Canizalez-Roman A, Gonzalez-Nuñez E, Vidal JE, Flores-Villaseñor $H$, León-Sicairos N. Prevalence and antibiotic resistance profiles of diarrheagenic Escherichia coli strains isolated from food items in northwestern Mexico. Int J Food Microbiol. 2013 Jun; 164(1):36-45.

30 Otero V, Rodríguez-Calleja JM, Otero A, GarcíaLópez ML, Santos JA. Genetic characterization of atypical enteropathogenic Escherichia coli isolates from ewes' milk, sheep farm environments, and humans by multilocus sequence typing and pulsedfield gel electrophoresis. Appl Environ Microbiol. 2013 Oct;79(19):5864-9.

31 Sydow ACMV, Coogan DG, Moreno JA, Melville AM, Benites NR. Ocorrência de fatores de virulência em estirpes de Escherichia coli isoladas de fezes de cães errantes. Arq Inst Biol. 2006 out-dez;73(4):401-7.

32 Caliman MCW. Estudo de vigilância bacteriológica: isolamento, fatores de virulência e resistência antimicrobiana de cepas de Escherichia coli isoladas de gatos domésticos na região de Ribeirão Preto [dissertação]. Jaboticabal (SP): Universidade Estadual Paulista Júlio de Mesquita Filho; 2010. 113 p.

33 Pereira CS, Barros PR, Silva PM, Rodrigues DP. Patógenos isolados do trato gastrintestinal de cães saudáveis no Rio de Janeiro. Arq Bras Med Vet Zootec. 2009 Aug;61 (4):1000-1.

34 Verdier K, Nyman A, Greko C, Bengtsson B. Antimicrobial resistance and virulence factors in Escherichia coli from Swedish dairy calves. Acta Vet Scand. 2012 Jan;54(2): 1 - 10.

35 Tóth I, Schmidt H, Kardos G, Lancz S, Creuzburg K, Damjanova I, et al. Virulence genes and molecular typing of different groups of Escherichia coli 0157 strains in cattle. Appl Environ Microbiol. 2009 Oct;75(19):6282-91.

36 Knöbl T, Godoy SN, Matushima ER, Guimarães MB, Ferreira AJP. Caracterização molecular dos fatores de virulência de estirpes de Escherichia coli isoladas de papagaios com colibacilose aviária. Braz J Vet Res Anim Sci. 2008;45 Supl:54-60.

37 Carvalho MV, Giles LC, Ziebell K, Ribeiro AM, CatãoDias JL, Sinhorini LI, et al. J Clin Microbiol. 2003 Mar;4 1 (3): 1225-34.

38 Förster M, Klimpel S, Mehlhorn H, Sievert K, Messler S, Pfeffer K. Pilot study on synanthropic flies (E.G. Musca, Sarcophaga, Calliphora, Fannia, Lucilia, Stomoxys) as vectors of pathogenic microorganisms. Parasitol Res. 2007 Jun;101(1):243-6. 
39 Cookson AL, Cao M, Bennett J, Nicol C, ThomsonCarter F, Attwood GT. Relationship between virulence gene profiles of atypical enteropathogenic Escherichia coli and Shiga toxin-producing E. coli isolates from cattle and sheep in New Zealand. Appl Environ Microbiol. 2010 Jun;76(1 1):3744-7.

40 Fröhlicher E, Krause G, Zweifel C, Beutin L, Stephan R. Characterization of attaching and effacing Escherichia coli (AEEC) isolated from pigs and sheep. BMC Microbiol. 2008 Sep;8(144): 1-6.

41 Oh JY, Kang MS, Hwang HT, An BK, Kwon JH, Kwon YK. Epidemiological investigation of eaeA-positive Escherichia coli and Escherichia albertii strains isolated from healthy wild birds. J Microbiol. 2011 Oct;49(5):747-52.

42 Oh JY, Kang MS, An BK, Shin EG, Kim MJ, Kim YJ, et al. Prevalence and characteristics of intimin-producing Escherichia coli strains isolated from healthy chickens in Korea. Poult Sci. 2012 Oct;91 (10):2438-43.

43 Chandran A, Mazumder A. Prevalence of diarrheaassociated virulence genes and genetic diversity in Escherichia coli isolates from fecal material of various animals hosts. Appl Environ Microbiol. 2013 Dec;79(23):7371-80.

44 Beaudry M, Zhu C, Fairbrother JM, Harel J. Genotypic and phenotypic characterization of Escherichia coli isolates from dogs manifesting attaching and effacing lesions. J Clin Microbiol. 1996 Jan;34(1):144-8.

45 Monaghan A, Byrne B, Fanning S, Sweeney T, Mcdowell D, Bolton DJ. Serotypes and virulence profiles of atypical enteropathogenic Escherichia coli (EPEC) isolated from bovine farms and abattoirs. J Appl Microbiol. 2013 Feb; 1 14(2):595-603.

46 Ibenyassine K, AitMhand R, Karamoko Y, Cohen $N$, Ennaji MM. Use of repetitive DNA sequences to determine the persistence of enteropathogenic Escherichia coli in vegetables and in soil grown in fields treated with contaminated irrigation water. Lett Appl Microbiol. 2006 Nov;43(5):528-33.

47 Sidhu JP, Ahmed, W, Hodgers L, Toze S. Occurrence of virulence genes associated with diarrheagenic pathotypes in Escherichia coli isolates from surface water. Appl Environ Microbiol. 2013 Jan;79(1): 328-35.

48 Albertini LS. Ecologia, fatores associados à virulência e diversidade de Escherichia coli isoladas de amostras de água de lastro, água de regiões portuárias e moluscos bivalves no Brasil [tese]. São Paulo (SP): Universidade de São Paulo; 2009. $215 \mathrm{p}$.

49 Melo SK. Caracterização de fatores de virulência em amostras de Escherichia coli isoladas de lagoas do Parque Estadual Rio Doce, Minas Gerais [dissertação]. Ouro Preto (MG): Universidade Federal de Ouro Preto; 2006. 100 p.
50 Orlandi PP, Magalhães GF, Matos NB, Silva T, Penatti M, Nogueira PA. Etiology of diarrheal infections in children of Porto Velho (Rondônia, Western Amazon region, Brazil). Braz J Med Biol Res. 2006 Apr;39(4):507-1.

51 Bueris V, Sircili MP, Taddei CR, Santos MF, Franzolin MR, Martinez MB, et al. Detection of diarrheagenic Escherichia coli from children with and without diarrhea in Salvador, Bahia, Brazil. Mem Inst Oswaldo Cruz. 2007 Nov; 102(7):839-44.

52 Oliva CAG, Scaletsky I, Morais MB, Fagundes Neto U. Diarréia aguda grave associada à Escherichia coli enteropatogênica clássica (EPEC): características clínicas e perdas fecais em lactentes hospitalizados. Rev Assoc Med Bras. 1997 out-dez;43(4):284-9.

53 Afset JE, Bevanger L, Romundstad P, Bergh K. Association of atypical enteropathogenic Escherichia coli (EPEC) with prolonged diarrhea. J Med Microbiol. 2004 Nov;53(Pt 1 1):1137-44.

54 Robins-Browne RM, Bordun AM, Tauschek M, Bennett-Wood VR, Russell J, Oppedisano F, et al. Escherichia coli and community acquired gastroenteritis, Melbourne, Australia. Emerg Infect Dis. 2004 Oct;10(10):1797-805.

55 Jafari A, Aslani MM, Bouzari S. Escherichia coli: a brief review of diarrheagenic pathotypes and their role in diarrheal diseases in Iran. Iran J Microbiol. 2012 Sep;4(3): 102-17.

56 Araujo JM, Tabarelli GF, Aranda KRS, Fabbricotti $\mathrm{SH}$, Fagundes-Neto $U$, Mendes CMF, et al. Typical enteroaggregative and atypical enteropathogenic types of Escherichia coli are the most prevalent diarrhea-associated pathotypes among Brazilian children. J Clin Microbiol. 2007 Oct;45(10):3396-9.

57 Bendezú RYA, Huasasquiche JGL. Serotipificación de Escherichia coli enteropatógena (EPEC) en cuadros diarreicos agudos de niños menores de cinco años: Hospital Nacional Docente MadreNiño San Bartolomé, noviembre 2000-marzo 2001 [tese]. Lima: Universidad Nacional Mayor de San Marcos; 2007.

58 Afset JE, Bergh KR, Bevanger L. High prevalence of atypical enteropathogenic Escherichia coli (EPEC) in Norwegian children with diarrhoea. J Med Microbiol. 2003 Nov;52(1 1):1015-9.

59 Blanco M, Blanco JE, Dahbi G, Mora A, Alonso MP, Varela $G$, et al. Typing of intimin (eae) genes from enteropathogenic Escherichia coli (EPEC) isolated from children with diarrhoea in Montevideo, Uruguay: identification of two novel intimin variants $(\mu \mathrm{B}$ and $\xi R / \beta 2 B)$. J Med Microbiol. 2006 Sep;55(9): 11 65-74. 
60 Costa ARF, Lima KVB, Souza CO, Loureiro ECB. Desenvolvimento de PCR multiplex para detecção e diferenciação de categorias de Escherichia coli diarreiogênicas. Rev Pan-Amaz Saude. 2010 jun; 1 (2):77-84.

61 Sousa EB. Aspectos microbiológicos e epidemiológicos da doença diarreica aguda no município de Juruti, Pará [dissertação]. Belém (PA): Universidade Federal do Pará, Instituto de Ciências Biológicas; 2010. 78 p.

62 Shetty VA, Kumar SH, Shetty AK, Karunasagar I, Karunasagar I. Prevalence and characterization of diarrheagenic Escherichia coli isolated from adults and children in Mangalore, India. J Lab Physicians. 2012 Jan-Jun;4(1):24-9.

63 Hannaoui E, Villalobos L, Martínez R, Maldonado A, Hagel I, Bastardo J. Diarrheagenic Escherichia coli associated with acute diarrhea in children of Cumaná, Venezuela. Invest Clin. 2010 Dec;51 (4):489-500.

64 Spano LC, Sadovsky AD, Segui PN, Saick KW, Kitaga SM, Pereira FE, et al. Agespecific prevalence of diffusely adherent Escherichia coli in Brazilian children with acute diarrhea. J Med Microbiol. 2008 Mar; 57(P+ 3):359-63

65 Vilchez S, Reyes D, Paniagua M, Bucardo F, Möllby R, Weintraub A. Prevalence of diarrhoeagenic Escherichia coli in children from León, Nicaragua. J Med Microbiol. 2009 May; 58(5):630-7.

66 Franzolin MR, Alves RC, Keller R, Gomes TA, Beutin L, Barreto ML, et al. Prevalence of diarrheagenic Escherichia coli in children with diarrhea in Salvador, Bahia, Brazil. Mem Inst Oswaldo Cruz. 2005 Jul;100(4):359-63.

67 Sakkejha H, Byrne L, Lawson AJ, Jenkins C. An update on the microbiology and epidemiology of enteropathogenic Escherichia coli in England 2010-2012. J Med Microbiol. 2013 Oct; 62(10):1531-4.

68 Scaletsky ICA, Aranda KRS, Souza TB, Silva NP, Morais MB. Evidence of pathogenic subgroups among atypical enteropathogenic Escherichia coli strains. J Clin Microbiol. 2009 Nov; $47(11): 3756-9$.

69 Gomes TAT, Irino K, Girão DM, Girão VBC, Guth BEC, Vaz TMl, et al. Emerging enteropathogenic Escherichia coli strains? Emerg Infect Dis. 2004 Oct;10(10): $1851-5$.

70 Afset JE, Bruant G, Brousseau R, Harel J, Anderssen E, Bevanger $L$, et al. Identification of virulence genes linked with diarrhea due to atypical enteropathogenic Escherichia coli by DNA microarray analysis and PCR. J Clin Microbiol. 2006 Oct;44(10):3703-11.
71 Dulguer MV, Fabbricotti SH, Bando SY, MoreiraFilho CA, Fagundes-Neto U, Scaletesky ICA. Atypical enteropathogenic Escherichia coli strains: phenotypic and genetic profiling reveals a strong association between enteroaggregative $E$. coli heatstable enterotoxin and diarrhea. J Infect Dis. 2003 Dec;188(11):1685-94.

72 Lluque A, Mercado E, Riveros M, Alvarado L, Carlos E, Colichón ASE, et al. Comparación entre el diagnóstico serológico y el diagnóstico por reación en cadena de la polimerasa (PCR) para Escherichia coli enteropatogénica (EPEC). Rev Gastroenterol Peru. 2010 abr-jun;30(2):121-5.

73 Gillespie SH. Medical microbiology. São Paulo: Premier; 2006. p. 252-4.

74 Moreira FC, Vieira MAM, Ferreira AJ, Girão DM, Vaz TM, Rosa AC, et al. Escherichia coli strains of serotype 051 : H40 comprise typical and atypical enteropathogenic E. coli strains and are potentially diarrheagenic. J Clin Microbiol. 2008 Apr;46(4):1462-5.

75 Paiva FPT. Quorum sensing em Escherichia coli enteropatogênica atípica [dissertação]. São Paulo (SP): Universidade de São Paulo, Instituto Butantan; $2011.30 \mathrm{p}$.

76 Bueris V. Interação de Escherichia coli enteropatogênica (EPEC) que apresenta o padrão de adesão localizada-like com a célula epitelial in vitro [tese]. São Paulo (SP): Universidade de São Paulo, Instituto de Ciências Biomédicas; 2008. 131 p.

77 Finlay BB, Rosenshine I, Donnenberg MS, Kaper JB. Cytoskeletal composition of attaching and effacing lesions associated with enteropathogenic Escherichia coli adherence to HeLa cells. Infect Immun. 1992 Jun;60(6):254 1 -3.

78 Nataro JP, Kaper JB, Robins-Browne R, Prado V, Vial $P$, Levine MM. Patterns of adherence of diarrheagenic Escherichia coli to HEp-2 cells. Pediatr Infect Dis J. 1987 Sep;6(9):829-31.

79 Pedroso MZ, Freymuller E, Trabulsi LR, Gomes TA. Attaching-effacing lesions and intracellular penetration in HeLa cells and human duodenal mucosa by two Escherichia coli strains not belonging to the classical enteropathogenic $E$. coli serogroups. Infect Immun. 1993 Mar;61 (3): 1 152-6.

80 Scaletsky ICA, Pedroso MZ, Oliva CAG, Carvalho RLB, Morais MB, Fagundes-Neto UA. Localized adherence-like pattern as a second pattern of adherence of classic enteropathogenic Escherichia coli to HEp-2 cells that is associated with infantile diarrhea. Infect Immun. 1999 Jul;67(7):3410-5.

81 Abe CM, Trabulsi LR, Blanco J, Blanco M, Dahbi $G$, Blanco JE, et al. Virulence features of atypical enteropathogenic Escherichia coli identified by the eae $^{+}$EAF-negative $s t x^{-}$genetic profile. Diagn Microbiol Infect Dis. 2009 Aug;64(4):357-65. 
82 Andrade JRC. Invasion by EPEC. Rev Microbiol. $1996 ; 27(1 S): 63-6$.

83 Kimata K, Shima T, Shimizu M, Tanaka D, Isobe J, Gyobu Y, et al. Rapid characterization of pathogenic Escherichia coli by multiplex PCR. Microbiol Immunol. 2005 Jun;(6):485-92.

84 López-Saucedo C, Cerna JF, Villegas-Sepulveda $\mathrm{N}$, Thompson R, Velazquez FR, Torres J, et al. Single multiplex polymerase chain reaction to detect diverse loci associated with diarrheagenic Escherichia coli dispatches. Emerg Infect Dis. 2003 Jan;9(1):127-31.

85 Toma C, Lu Y, Higa N, Nakasone N, Chinen I, Baschkier A. Multiplex PCR assay for identification of human diarrheagenic Escherichia coli. J Clin Microbiol. 2003 Jun;41(6):2669-71.

86 Aranda KRS, Fabbricotti SH, Fagundes-Neto $U$, Scaletsky ICA. Single multiplex assay to identify simultaneously enteropathogenic, enteroaggregative, enterotoxigenic, enteroinvasive and Shiga toxin-producing Escherichia coli strains in Brazilian children. FEMS Microbiol Lett. 2007 Feb;267(2): 145-50.

87 Bouzari S, Aslani MM, Oloomi M, Jafari A, Dashti A. Comparison of multiplex PCR with serogrouping and PCR-RFLP of fliC gene for the detection of enteropathogenic Escherichia coli (EPEC). Braz J Infect Dis. 2011 Jul-Aug;15(4):365-9.

88 Novais CM, Alves MP. PCR em tempo real: uma inovação tecnológica da reação em cadeia da polimerase. Rev Biotecnol Cienc Desenvol. 2004 jul-dez;33: 10-3.

89 Bortolini MR, Trabulsi LR, Keller R, Frankel G, Sperandio V. Lack of expression of bundle-forming pili in some clinical isolates of enteropathogenic Escherichia coli (EPEC) is due to a conserved large deletion in the bfp operon. FEMS Microbiol Lett. 1999 Oct;179(1):169-74.

90 Ferrer SR. Fatores de risco das diarreias em crianças em Salvador, Bahia [tese]. Salvador (BA): Universidade Federal da Bahia; 2007. 385 p.
91 Sousa CP. Escherichia coli um patógeno bacteriano especializado. Rev Biol Cienc Terra. 2006;6(1): 341-52.

92 Yatsuyanagi J, Saito S, Miyajima Y, Amano K-I, Enomoto K. Characterization of atypical enteropathogenic Escherichia coli strains harboring the astA gene that were associated with a waterborne outbreak of diarrhea in Japan. J Clin Microbiol. 2003 May;41(5):2033-9.

93 Vieira MAM, Salvador FA, Silva RS, Irino K, Vaz TMI, Rockstroh AC, et al. Prevalence and characteristics of the 0122 pathogenicity island in typical and atypical enteropathogenic Escherichia coli strains. J Clin Microbiol. 2010 Apr;48(4): 1 452-5.

94 Elias WP, Barros SF, Moreira CG, Trabulsi LR, Gomes TAT. Enteroaggregative Escherichia coli strains among classical enteropathogenic Escherichia coli $\bigcirc$ serogroups. J Clin Microbiol. 2002 Sep;40(9):3540-1.

95 Bielaszewska M, Middendorf B, Köck R, Friedrich AW, Fruth A, Karch $H$. Shiga toxin-negative attaching and effacing Escherichia coli: distinct clinical associations with bacterial phylogeny and virulence traits and inferred in host-pathogen evolution. Clin Infect Dis. 2008 Jul;47(2):208-17.

96 Schmidt MA. LEE ways: tales of EPEC, ATEC and EHEC. Cell Microbiol. 2010 Nov; 12(11):1544-52.

97 Abreu AG, Bueris V, Porangaba TM, Sircili MP, Navarro-Garcia F, Elias WP. Autotransporter roteinencoding genes of diarrheagenic Escherichia coli are found in both typical and atypical enteropathogenic E. coli strains. Appl Environ Microbiol. 2013 Jan;79(1):411-4.

98 Vieira MAM, Andrade JRC, Trabulsi LR, Rosa ACP, Dias AMG, Ramos SRT, et al. Phenotypic and genotypic characteristics of Escherichia coli strains of non-enteropathogenic E. coli (EPEC) serogroups that carry EAE and lack the EPEC adherence factor and shiga toxin DNA probe sequences. J Infect Dis. 2001 Mar;183(5):762-72. 